\title{
La clientela nelle Satire di Giovenale
}

\author{
MÔNICA COSTA VITORINO \\ Departamento de Letras Clássicas \\ Faculdade de Letras da Universidade Federal de Minas Gerais
}

\begin{abstract}
RESUMO: Este artigo tem como objetivo analisar as relações entre patronos e clientes, um tema amplamente tratado nas sátiras de Juvenal e que constitui um instrumento extremamente importante para o estudo do retrato da sociedade romana fornecido pelo satírico.
\end{abstract}

PALAVRAS CHAVE: Juvenal; sátira; cliente; patrono; sociedade romana.

L'opera di Giovenale offre abbondantemente elementi e testimonianze che vanno oltre la sfera della vita privata stricto sensu, potendo costituire in realtà dati rilevanti per una storia sociale dell'impero romano. Dal punto di vista scientifico però le informazioni fornite dall'autore devono essere utilizzate con la dovuta cautela, giacché molte volte tali informazioni non si accordano del tutto con i dati offerti dalle fonti storiche tradizionali. Innanzitutto si deve osservare che Giovenale non è uno scrittore di storia, ma un poeta che sceglie la società del suo tempo come argomento privilegiato. Egli non parte dal punto di vista dello storico, che osserva il funzionamento delle istituzioni civili e militari e gli avvenimenti della vita politica; il suo punto di vista è piuttosto quello interno alla società stessa, quello di un comune cittadino. Inoltre per essere un poeta satirico, Giovenale, d'accordo con il proprio programma poetico, dà maggior rilievo a determinati aspetti della società che desidera criticare e, perciò, soprattutto riguardo agli esempi utilizzati dall'autore, è necessario tener in conto la deformazione ed esagerazione intenzionali che, letterariamente, egli mette a frutto.

La clientela, come istituzione sociale, è uno degli argomenti più oscuri della storia romana. Giovenale, sebbene scriva satira, è tra le fonti più importanti per lo studio di questa istituzione ${ }^{1}$. Fonte preziosa per le informazioni riguardanti la clientela all'inizio del II secolo a.C. è costituita da alcune commedie di Plauto ${ }^{2}$, ma gli elementi costitutivi del rapporto

Secondo N. Rouland (Rouland: 1979, p. 514), une étude de la pratique clientélaire et des groupes sociaux que s'y adonaiant est donc possible, sourtout si l'on compléte par le recours à quelques autres sources les renseignements donnés par Martial et Juvénal.

2 Le commedie di Plauto non hanno carattere giuridico, ma i testi descrivono situazioni che possono essere considerate come situazioni quotidiane autentiche, costituendo uma testimonianza abbastanza fedele della vita sociale all'epoca dell'autore. 
clientelare si sono però abbastanza modificati con l'ascensione del nuovo regime. Dalla descrizione che si trova in Plauto, si può verificare che la situazione della clientela descritta da Giovenale è radicalmente diversa. Fra alcune delle trasformazioni avvenute, si osseva che mentre il comediografo diceva che al tempo suo tutti volevano il maggior numero possibile di clienti, volendo con ciò affermare il loro valore e la loro forza come gruppo sociale, il satirico afferma:

...Nusquam minor est iactura clientis (III, 125)

in nessun'altra occasione

è più facile cacciare un cliente ${ }^{3}$.

Tra i diversi argomenti di natura sociale presenti nelle satire di Giovenale, il rapporto tra clienti e patrono è senza dubbio il più ricorrente e quasi sempre visto da un'inquadratura soprattutto economica. Il quadro presentato nell'insieme è il ritratto di una crisi. Nell'opinione del satirico, a guastare tale rapporto, con gravi conseguenze sull'intera società, sta quello che l'autore identifica come il grande problema del tempo: l'auaritia. Questa si manifesta in ambedue le parti che istituiscono il rapporto clientelare, sotto forma di avarizia dalla parte dei patroni, che si negano a retribuire giustamente il cliente con sportule e cene più degne di un amico, sia dalla parte dei clienti, troppo interessati ai guadagni provenienti dalla sportula e alla possibilità di diventare eredi dei loro patroni.

In una società come quella descritta da Giovenale non c'è più spazio per il rispetto 0 per l'amicizia. Da parte dei patroni gli obblighi verso la società imposti dal loro rango sociale sembrano essere allora del tutto spariti, e ciò che rimane del loro orgoglio di appartenere alle classi più alte della società resta legato alla semplice ostentazione esteriore delle ricchezze possedute. Alla moltiplicazione di ville sontuose e alla diffusione dell'abitudine di cene favolose mangiate da soli si contrappone la piccola sportula (sportula [...] parua: I, 95-96). Questa, secondo Giovenale, non verrebbe contesa soltanto dalla folla di umili clienti, ma persino da alti magistrati.

I patroni non si curano delle necessità dei vecchi clienti che pure sono sempre lì a rendere i loro servigi. Nelle satire si riprende il tema già così comune nelle commedie di Plauto: i patroni si interessano piuttosto dei clienti ricchi, che possono rappresentare per loro qualche speranza di guadagno. Insomma non c'è più posto per la lealtà o la virtù di un cliente povero e trovare queste qualità in un ricco sarebbe proprio impossibile; chi comanderebbe nella Roma di allora sarebbe prima di tutto il denaro, la principale fonte della corruzione e dei vizi.

La giornata del cliente viene descritta da Giovenale come ordinata in una routine molto ben definita: prima la sportula, chiaramente associata dall'autore alla salutatio mattinale, poi la dedizione a quelle attività propriamente legate alla vita pubblica e al foro:

3 I brani sono stati trascritti secondo il testo stabilito da W. V. Clausen pubblicato dalla Oxford University Press, uso le traduzioni di L. Paolicchi, pubblicata dalla Salerno Editrice. 
ipse dies pulchro distinguitur ordine rerum:

sportula, deinde forum... (I, 127-128)

La giornata é ben ordinata in una serie di cose:

la sportula, poi il Foro...

Il poeta non fa menzione degli impegni del pomeriggio del cliente, probabilmente in parte dedicato al bagno nelle terme, ma non tace sulle opinioni dei clienti sul coronamento più felice della giornata, che sarebbe la cena con il patrono. Per il cliente povero un tale invito arriva soltanto molto raramente:

uestibulis abeunt ueteres lassique clientes

uotaque deponunt, quamquam longissima cenae

spes homini... (I, 132-143)

I vecchi e stanchi clienti lasciano i vestiboli

e abbandonano il sogno,

per quanto sia in un uomo ostinata

la speranza di una cena...

Giovenale e Marziale sono tra le fonti più importanti per la conoscenza della situazione della clientela al tempo dell'impero, principalmente per la questione della sportula, ma gli studiosi moderni hanno rilevato alcune discrepanze tra i due autori, le quali possono essere riunite in due punti principali: 1) Marziale parla della distribuzione della sportula verso la sera, dopo il bagno (Marz., III,7; X,70); ciò presuppone la connessione originale con la cena; Giovenale invece la colloca nella mattina, associandola con la salutatio; 2) Giovenale rappresenta nobili, magistrati, uomini liberi ricchi e anche donne che ricevono la sportula; questo non appare affatto in Marziale.

Riguardo a ciò, l'opinione di J. Le Gall (Le Gall, 1966, p. 1449-53) è abbastanza radicale. In un articolo molto succinto e breve in confronto alla complessità dell'argomento, egli afferma che la nuova plebe non conobbe queste distribuzioni quotidiane di sportule come descritte da Giovenale e Marziale; H. Stephenson (Stephenson, 1887, p. 243), anch'egli in un articolo brevissimo ma ancora importante, è d'accordo con la descrizione di Marziale e afferma che la pratica sociale romana alleata al silenzio di altre fonti dimostra l'improbabilità della presenza di donne nelle salutationes, così come il ricevimento della sportula da parte loro. J. Gérard (Gérard, 1976, p. 180) crede invece che queste indicazioni diverse non siano contraddittorie e dimostrino infatti l'esistenza di una certa libertà nelle abitudini proprie a ogni casa.

Per superare tali discrepanze, E. Courtney (Courtney, 1980, p. 105) propone le seguenti possibili soluzioni. Riguardo al primo punto, gli autori potrebbero riferirsi a due gruppi diversi: Marziale agli anteambulones, cioè quei clienti che accompagnavano il patrono per l'intera giornata e che ricevevano quindi la loro sportula alla fine delle loro prestazioni; Giovenale dovrebbe quindi riferirsi a quei clienti che vedevano il patrono soltanto durante la salutatio mattinale. Quanto al secondo punto, lo studioso suppone che Giovenale 
abbia volontariamente soppresso la vera natura dei rapporti tra un uomo prominente e un altro personaggio di rango anche alto però non tanto come il suo; un tale personaggio dovrebbe venire comunque a salutare un altro personaggio superiore della propria condizione, anche se non aveva con questi un rapporto di clientela. Il fatto che accettava di ricevere la sportula era il compimento di una mera formalità e questa doveva essere ripartita ai suoi accompagnatori; secondo lo studioso, Giovenale userebbe questa specie di malinteso per amplificare l'indignatio della composizione.

Restando però al testo di Giovenale, non si può assolutamente parlare della soppressione di un dato reale in nome di un'amplificazione della forza persuasiva della satira perché l'affermazione del satirico che anche il cliente ricco riceve la sportula è fondamentale a tutta la sua critica nell'avidità dei ricchi, e inoltre tale affermazione viene posteriormente esplicitamente rafforzata:

sed cum summus honor finito conputet anno, sportula quid referat, quantum rationibus addat... (I, 117-118)

Ma se, a fine anno, i più alti magistrati fanno il conto di quanto la sportula rende, quanto aggiunge agli stipendi...

La presenza di cittadini di alto rango tra la folla di clienti viene, anzi, diverse volte riaffermata dall'autore. La presenza di questi elementi di alta classe nella salutatio e conseguentemente nella distribuzione della sportula sarebbe dovuta, secondo il parere di Le Gall (Le Gall, 1971, p. 270), alla necessità di guadagnare elettori per le dispute che ancora erano in vigore, con il fine di ottenere il fascium decus et imperium. Siccome la vita politica si stava concentrando sempre più nelle mani del princeps, più che una carriera specificamente politica sarebbe la nomina a posti amministrativi oppure una promozione nel cursus honorum ciò che potrebbe essere ottenuto dal patrono in beneficio dei suoi clienti. Secondo il Rouland (Rouland, 1979, p. 520), questo genere di servizio sarebbe evidentemente fornito a quei clienti che avrebbero già raggiunto un certo rango sociale ma che, giustamente perché mossi da questo interesse, prendevano parte alla salutatio mattinale e alla distribuzione della sportula. Nella distribuzione della sportula, secondo Giovenale, si doveva osservare una certa gerarchia: ad essa facevano capo i discendenti delle grandi famiglie romane e anche questi nell'ordine del loro rango sociale:

...iubet a praecone uocari

ipsos Troiugenas, nam uexant limen et ipsi

nobiscum. "da praetori, da deinde tribuno." (I, 99-101)

Ordina al banditore di fare l'apello

anche dei discendenti di Troia,

perché, con noi, anche loro fanno ressa alla soglia.

"Dài al pretore, poi al tribuno". 
Ma non è questo il fatto che provoca di più l'indignazione del poeta, bensì la sovversione dell'ordine sociale tradizionale a causa dall'avidità di denaro. Poiché un liberto, pur se venuto a Roma come schiavo, una volta arricchitosi abbastanza, può rivendicare una posizione superiore a quella degli autentici discendenti degli antichi romani basandosi semplicemente sul suo reddito, arrogandosi persino i diritti di un cavaliere, soltanto perché le loro quinque tabernae quadrigenta parant (I, 105-106). La porpora deve quindi cedere la precedenza al denaro.

L'attaccamento ai soldi ottenuti con la sportula non viene tanto condannato per $\mathrm{i}$ clienti poveri che di lì ricavano la propria sussistenza, ma principalmente per coloro che, pur potendo prescindere da tale beneficio, neanche si preoccupano per le umiliazioni a cui si espongono per avere questa fonte di reddito. Sono piuttosto queste persone quelle che Giovenale vuole attaccare e criticare quando tratta l'argomento della sportula. Non c'è dunque ragione per credere che tutti quei ricchi di cui parla il poeta siano soltanto personaggi inventati, giacché ciò svuoterebbe tutta la satira di qualsiasi reale impegno verso la società.

In quello che riguarda particolarmente la presenza di donne in uno o altro polo del legame di clientela, la situazione assume sfumature alquanto diverse. L'esistenza di matrone le quali hanno clienti di alto e di basso rango sociale e che vanno da loro per la salutatio mattinale, tutti mossi da interessi più o meno venali, è affermata esplicitamente:

quod porro officium, ne nobis blandiar, aut quod
pauperis hic meritum, si curet nocte togatus
currere, cum praetor lictorem inpellat et ire
praecipitem iubeat dudum uigilantibus orbis,
ne prior Albinam et Modiam collega salutet? (III, 126-130)
D'altra parte, per non farci illusioni,
che vale qui l'ossequio, il merito d'un cliente
che si dia pensiero di correre al saluto,
quando ancora è notte, vestito di toga,
se un pretore manda il littore
e gli comanda di correre svelto,
nel caso le vedove senza figli siano già sveglie,
perché, a salutare Albina e Modia,
non arrivi prima il collega?

In questo contesto l'esistenza di clienti ricchi appare più verosimile. Il vero interesse di questi clienti non potrebbe realmente essere la modesta somma rappresentata dalla sportula, invece la possibilità di essere citati in testamento da una vedova ricca $e$ senza figli potrebbe rappresentare un'attrattiva più consistente. Questa possibilità si verificherebbe ugualmente nel caso di un riccone senza eredi diretti (VI, 548-549; XII, 99101) e costituirebbe anch'essa comunque una forma di captatio, essendo quindi una motivazione assai plausibile per l'esistenza di quegli aristocratici salutatores, mossi da interessi pecuniari, di cui parla Giovenale. Quanto alla presenza di donne tra questi 
salutatori, ricevendo anch'esse la sportula, il fatto viene accennato dal poeta riferendosi però soltanto alle mogli dei clienti ricchi:

\section{...sequiturque maritum}

languida vel praegnas et circumducitur uxor.

hic petit absenti nota iam callidus arte

ostendens uacuam et clausam pro coniuge sellam.

"Galla mea est" inquit, "citius dimitte. moraris?

profer, Galla, caput. noli uexare, quiescet." (I, 121-126)

...segue il marito nel giro.

Uno, esperto nel vecchio trucco,

chiede per la moglie che non c'è,

mostrando, invece, della moglie,

la portantina vuota e chiusa.

"C'è la mia Galla - dice - mandami via più presto.

Non ci crede? Galla metti fuori la testa.

Non la disturbare, forse dorme".

Se si accetta un minimo di verosimiglianza nella composizione della satira, si deve credere che almeno le mogli dei clienti benestanti potrebbero ricevere la sportula, una volta che l'elemento di assurdità della scena rappresentata dal poeta satirico e che suscita il suo sdegno non sia tanto il fatto di una donna che riceve la sportula, ma sì il fatto che una moglie pure ammalata o incinta voglia seguire il marito dappertutto e, principalmente, che il marito, anche assente la moglie, mentendo sulla sua assenza chieda la sportula anche per lei.

Anche qui, pur ammettendo il carattere eccezionale e assurdo della situazione rappresentata, si deve presupporre che una scena così dettagliata debba corrispondere a un uso sociale consueto, giacché viene presentata come una consuetudine del tutto naturale. Secondo il Gérard (Gérard, 1976, p. 163), una falsificazione sulle abitudini nei rapporti sociali del tempo a questo punto farebbe dimminuire ogni livello di credibilità sulla realtà contemporanea che la satira, come genere letterario, intende affrontare.

Un altro elemento importante riguardante quella clientela di alto rango sociale che si può ricavare dal testo delle Satire è l'accenno alla possibilità dell'ossequio a diversi patroni. Nei due brani appena citati, le parole orbis e circumducitur sembrano dimostrare l'esistenza di un vero percorso di visite. I clienti, quindi, sembrano aver potuto avere più di un patrono, o matrona come nel primo dei brani dove vengono citati i nomi di Albina e di Modia (I, 130). Tale ipotesi sembra poter essere estesa anche ai clienti poveri: nella satira V si dice: tota salutatrix iam turba peregerit orbem... (V, 21); infatti il basso valore della sportula, di cui si parlerà più avanti, presupporrebbe la necessità di questo espediente per la sussistenza dei clienti poveri, mentre per i ricchi diventerebbe più plausibile l'affermazione contenuta nei versi I, 117-118, già citati, secondo i quali anche i magistrati alla fine dell'anno facevano $i$ conti di quanto avevano reso loro i soldi della sportula. 
Il termine sportula, preso alla lettera, significa "cestino". Secondo il Courtney (Courtney, 1980, p. 104), alle origini dell'istituzione clientelare, un cliente poteva aspettarsi dal patrono in retribuzione ai servizi prestati un invito a cena; ma con l'aumento del numero di clienti alla fine tale consuetudine diviene impraticabile. Allora al posto della cena si istituisce l'abitudine di distribuire cibo, da portare a casa, in piccoli cesti, appunto in sportulae. Più tardi a queste sportulae contenenti generi alimentari si sostituisce una certa quantità di soldi. Nelle Satire di Giovenale il termine sportula appare in tre significati diversi: 1) come la somma di denaro distribuita ai clienti, così in I, 95; 118; 128; X, 46;2) con un'allusione oscura, generalmente intesa come una specie di picnic, in III, 249; 3) per indicare i clienti stessi, in XIII, 33. Non appare quindi nessun riferimento al significato originale della sportula come un cestino di alimenti, un uso probabilmente già sparito da molto tempo all'epoca in cui visse il poeta.

Da un'informazione di Svetonio (Dom., 7, 1), è saputo che Domiziano aveva abolito negli intrattenimenti pubblici la distribuzione di denaro, ristaurando al suo posto l'uso della cena. In questo, secondo il Courtney (Courtney, 1980, p. 104-5), il Paolicchi (Paolicchi, 1996, p. 165-6) e il Gérard (Gérard, 1976, p. 178-179), l'imperatore fu seguito per qualche tempo dai patroni privati, ma presto si ritornò all'uso anteriore e sotto Traiano la sportula, come mancia in denaro, è ufficialmente ritornata, con il valore fisso di cento quadranti. Questo valore è riportato sia da Marziale, Sportula non iunxit quadrantibus arida centum (Marz., X, 75, 11), sia da Giovenale: ...densissima centum quadrantes lectica petit... (I. 120-121). Entrambi gli autori si preoccupano di far risaltare l'esiguità di tale somma. Giovenale oppone, non senza ironia, il cliente povero, che deve ricavare dalla sportula la toga, le scarpe, il pane e il fumo (non il calore) del focolare: (I, 119-120), al cliente ricco che però non la disprezza affatto. Marziale a sua volta parla dell'impossibilità della sopravvivenza del cliente con un sussidio così irrisorio.

Tornando al valore della sportula, i cento quadranti corrisponderebbero a 25 assi, cioè a 6,25 sesterzi. Un cliente assiduo poteva dunque ricavare con la sportula offerta dal patrono una somma di circa 2,2 mila sesterzi all'anno. Un totale che è certamente troppo esiguo se comparato ai quattrocento mila sesterzi richiesti dal censo dei cavalieri, ritenuti da Giovenale stesso come un limite equilibrato all' ambizione di un uomo (XIV, 322-324). In realtà, con la somma necessaria per ottenere l'ammissione all'ordine equestre, un cittadino poteva procurarsi un reddito fisso che non sarebbe neanche dieci volte superiore a quel reddito che potrebbe provenire dalla sportula. Venti mila sesterzi sono infatti quanto chiede Nevolo, nella satira IX, alla Fortuna: uiginti milia fenus, pigneribus positis... (IX,140-141).

Secondo il Carcopino (Carcopino, 1994, p. 81) e il Bellandi (Bellandi, 1974, p. 288, n. 18), l'interesse normale, il fenus, al tempo di Giovenale era di 5\%. Il Courtney (Courtney, 1980, p. 443) comparando la somma con i 24.000 sesterzi all'anno di Marziale, III, 10, ossia il $6 \%$, dice che Nevolo, volendo $5 \%$, sarebbe stato contento con un interesse più modesto. Ma, dalla stessa satira, sembra essere possibile affermare che, oltre la solita sportula, il cliente poteva ricevere dal patrono, in beni o in denaro, altre ricompense per le sue prestazioni, anche se queste, come nel caso di Nevolo, di cinque mila sesterzi all'anno, non siano d'accordo con i molti lavori prestati:

computat et ceuet. Ponatur calculus, adsint cum tabula pueri; numera sestertia quinque omnibus in rebus, numerentur deinde labores. (IX,40-42) 
Facciamo il conto, vengano i servi con l'àbaco.

In tutto conta cinquemila sesterzi, poi facciamo il conto delle fatiche.

Parlando della distribuzione della sportula, le espressioni adoperate da Giovenale, come turba togata (I, 96) e densissima lectica (I, 120-121), mettono sempre in risalto la grande quantità di persone, siano ricchi siano poveri, che si presentano per ricevere l'elargizione. Dal punto di vista del patrono quindi la distribuzione quotidiana della sportula a un numero considerevole di clienti doveva rappresentare un esborso parimenti considerevole. Facendo i conti, un patrono che, per esempio, distribuiva ogni giorno i venti cinque assi della sportula a cento clienti (un numero suggerito da III, 229: centum Pythagoreis; oppure da III, 250: centum convivae) avrebbe dovuto spendere con la sportula alla fine di un anno una somma superiore a duecento mila sesterzi, equivalente alla metà del reddito esatto dal censo di un cavaliere, una somma dunque non del tutto irrilevante. Su questo, il Le Gall (Le Gall, 1971, p. 272) fa la seguente affermazione: Les patrons étaient peut-être fiers d'avoir de nombreux clients; on comprend cependant que beaucoup en aient limité le nombre.

Il punto di vista di Giovenale però non si avvicina mai a quello del patrono. Contro la mancata liberalità dei ricconi dell'epoca verso i loro dipendenti, l'autore pone la loro prodigalità nella costruzione di numerose e lussuose ville (XIV, 86-95) e le grandi somme perse nel gioco (I, 92-93). Tutto sommato il patrono è visto, principalmente riguardo al cliente povero, come un avaro, e le sue preoccupazioni con i soldi spesi nella distribuzione della sportula, che l'autore considera meschine, vengono messe in ridicolo:

ille tamen faciem prius inspicit et trepidat ne suppositus uenias ac falso nomine poscas:

agnitus accipies... (I, 97-99)

Ma prima lui ti guarda in faccia:

teme che tu venga al posto di un' altro

e chieda sotto falso nome.

La riceverai una volta riconosciuto.

In questi versi, che rispechiano le preoccupazioni di contabilità del patrono, si può intravedere che l'istituzione della clientela, o almeno la cerimonia della salutatio e l'elargizione della sportula, mantiene ancora, anche se forse solo superficialmente, il carattere di rapporto personale, basandosi su una sufficiente conoscenza reciproca delle parti. Alcuni studiosi (e.g. Highet, 1960, p. 7) credono che la sostituzione progressiva del pasto offerto dal patrono con la sportula in denaro testimonierebbe la perdita del caratere personale, quasi patriarcale che la relazione di clientela ancora conservava. A questo proposito il Rouland (Rouland, 1979, p. 529) puntualizza che le variazioni nelle modalità della distribuzione della sportula non procedono dalla volontà degli uomini potenti bensì da quella del princeps. La questione possiede un aspetto politico e si integra nel quadro di un conflitto latente tra il princeps e le clientele dei grandi uomini del tempo, di cui egli cerca di limitare le dimensioni. 
Per mezzo delle affermazioni di Giovenale si è consapevole che quei valori che originalmente regolavano il rapporto clientelare erano allora messi in crisi. Valori come la capacità personale, l'onore e la lealtà sono soppiantati dagli interessi economici. La critica all'auaritia dei patroni riguarda particolarmente il fatto che questi non ci tengono più all' amicitia, che dovrebbe essere il vincolo principale dei rapporti tra clienti e patroni (cf. Rouland, 1979, p. 573-77; Saller, 1989, p. 49-61). Dal rapporto presentato dall'autore emerge che gli uomini potenti vogliono consolidare la loro posizione sociale e la loro popolarità senza nessuna concessione di liberalità verso i loro clienti.

La popolarità, come tutto a Roma, può essere facilmente comprata. Il lungo corteggio di niueos Quirites che accompagnano il pretore nella processione di apertura dei giochi del circo, la cui amicizia è stata comprata con la sportula (X, 44-46), ne è un esempio. Altro esempio gli applausi nel tribunale, anch'essi comprati:

\section{nos hominum diuomque fidem clamore ciemus}

quanto Faesidium laudat uocalis agentem

sportula?... (XIII, 31-33)

E noi invochiamo l'aiuto di uomini e dei

con grido pari a quello dei clienti

che acclamano Fesidio in tribunale?

Secondo il Le Gall (Le Gall, 1971, p. 269) la sportula, come un piccolo stipendio, irregolarmente fornito, costituito da una somma in denaro, avrebbe un significato derivato dal senso primitivo di sportula, come un piccolo cesto usato per trasportare alimenti. Questo stipendio era dato a coloro che facevano parte del lungo corteggio di un magistrato $(X, 45-46)$ nell'occasione in cui questi offriva i giochi al pubblico. Tale era anche la sportula che il manceps, capo della claque, offriva ai suoi uomini dopo che essi avevano finito di applaudire l'avvocato che aveva loro prestato assistenza (XIII, 31-32). Comunque coloro che applaudivano dietro pagamento non dovevano essere così numerosi quanto si potrebbe credere, giacché essi potevano passare molto facilmente da un processo ad altro durante lo stesso giorno.

Un grande numero di clienti doveva certamente essere un elemento importante per l'incremento del prestigio personale e politico di un uomo potente. La folla che ogni mattina si raduna nel vestibolo della sua casa per la salutatio e quella che lo segue dappertutto nei suoi movimenti all'interno della città, ossia l'adsectatio, costituivano due forme di espressione del suo potere, forme repubblicane che si trovano praticamente ancora immutate in questo periodo. La propria struttura edilizia tipica della casa romana dev'essere vista nel contesto della struttura sociale delle attività alle quali essa doveva rispondere funzionalmente; su questo Wallace-Hadrill (Wallace-Hadrill, 1989, p. 63-64) afferma: the way the Roman house invites the viewer from the front door, unparalleled in the Greek world, flows from the patronal rituals so often described in the Roman sources: the opening of the doors at down to the crowd of callers, the acessibility of the dominus to the public, his clients and his friends (cf. anche Vitr., VI, 5, 1-2). Il vecchio cliente, certamente presente nel ricco vestibolo della casa del patrono per la salutatio, probabilmente, secondo ciò che si può cogliere nelle Satire, non partecipa alla sua comitiva. Umbricio si rammenta di non uscire mai in 
compagnia di nessuno (nulli comes exeo, III, 47) e Giovenale stesso, tra altre cose, indica come essenziale alla dimostrazione della perfetta nobiltà di un cittadino di alto rango avere un seguito irreprensibile (VIII, 127-133).

In questo quadro dovrebbe essere realmente piccolo l'interesse che un cliente potrebbe, personalmente, rappresentare per il patrono. Neanche in tribunale il povero potrebbe essere di effettivo aiuto al patrone, poiché secondo Giovenale non dovrebbe godere neppure di credibilità nel caso di essere presentato come teste in un processo. Allora a Roma reputazione e credito alla testimonianza dipenderebbero solamente della ricchezza:

quantum quisque sua nummorum seruat in arca, tantum habet et fidei... (III,143-144)

Quanto denaro ognuno ha nella sua cassaforte, tanto il credito che ha.

Agli occhi dei ricchi il povero è visto come del tutto inutile. Il cliente povero non conta niente in una società che lo disprezza e che ride della sua disgrazia (III, 147-151), ma egli, comunque, vuole farne parte e non intende rinunciare a quel poco di rispettabilità sociale che crede ancora di meritare. Ma tutti i suoi sforzi in questo senso sono però inutili. A Roma, secondo Giovenale, non ci sarebbe scampo per il povero. Questo non trovava altra fonte di reddito che la sportula e i servizi prestati al patrono i quali non venivano sufficientemente ricompensati. I prezzi altissimi della città rendevano impossibile vivere con una certa dignità e il cliente sarebbe quindi ridotto alla miseria, una volta che non ci fosse più posto per il lavoro onesto, degno di un uomo libero:

\section{... "quando artibus" inquit "honestis \\ nullus in urbe locus, nulla emolumenta laborum, res hodie minor est here quam fuit atque eadem cras deteret exiguis aliquid..." (III, 21-24).}

..."Visto che a Roma non c'è posto per mestieri onesti né compenso alle fatiche (quello che uno ha, oggi vale meno d'ieri, e domani scemerà di qualcosa dal poco che era)..."

Se si ricorda il fatto che nella società di quel tempo qualsiasi forma di lavoro manuale, e non solo, veniva considerata indegna di un uomo libero, e che quelle poche attività che questi poteva esercitare, come fare il maestro di grammatica o di retorica, oppure la professione di avvocato, sono dichiarate da Giovenale stesso come abbastanza mal ricompensate, le alternative che rimangono al cliente povero sono molto limitate. Questi, non possedendo beni o schiavi che gli possano assicurare un reddito regolare e sicuro, aveva un numero molto limitato di possibilità di risolvere i suoi problemi: quella di essere citato nel testamento di qualche ricco, quella di trovare un'occupazione rimunerata presso qualche magistrato e, infine, quella di avere i suoi servigi riconosciuti e venire gratificato bene dal patrono. 
Secondo Giovenale però tutte queste tre possibilità non erano altro che vane speranze. Quanto alle prime due, l'autore osserva:

...quis pauper scribitur heres?

quando in consilio est aedilibus?... (III, 161-162)

...Quale povero viene iscritto come erede?

Quando è chiamato nel consiglio per gli edili?...

Quanto alla speranza della gratitudine del patrono, questa si poteva realizzare soltanto nel caso del cliente abbandonando totalmente la sua dignità, trasformandosi in un adulatore, in un bugiardo, in un falso, oppure prestarsi a fare i lavori più sordidi, ma neanche in questi casi, come dimostra l'esempio di Nevolo, il cliente povero poteva aver la garanzia di un'effettiva ricompensa.

\section{Referências Bibliográficas}

BELLANDI, F. Naevolus cliens. Maia. Bologna, n. 26, p. 279-99, 1974.

CARCOPINO, J. La vita quotidiana a Roma. Roma-Bari: Laterza, 1994 .

CLAUSEN, W. V. A Persi Flacci et D. Iunii Iuvenalis Saturae. Oxford: Oxford University Press, $1988^{7}$.

COURTNEY, E. A Commentary on the Satires of Juvenal. London: The Athlone Press, 1980. GÉRARD, J. Juvénal et la réalité contemporaine. Paris: Les Belles Lettres, 1976.

HIGHET, G. Juvenal, the Satirist. Oxford: Clarendon Press, I960².

LE GALL, J. La "nouvelle plébe" et la sportule quotidienne. In CHEVALIER, R. (Ed.). Mélanges d'archeologie et d'histoire offerts à André Pignaiol. Paris: 1966, p. 1449-53. . Rome, ville de fainéants? Revue des Études Latines. Paris, n. 49, p. 226-77, 1971.

PAOLICCHI, L. (cur.), Persio - Giovenale, Le satire. Roma: Salerno Editrice, 1996.

ROULAND, N. Pouvoir politique et dépendance personelle dans l'antiquité romaine, Genèse et rôle des rapports de clientèle (Collection Latomus 166). Bruxelles: Société d'études latines de Bruxelles, 1979.

RUDD, N. Themes in Roman Satire. London: G. Duckworth, 1986.

SALLER, R.. Patronage and Friendship in Early Imperial Rome: Drawing the Distinction. In WALLACE-HADRILL, A. (Ed.). Patronage in Ancient Society. London-New York: Routledge, 1989, p. 49-62.

STEPHENSON, H. M. Difficulties in Juvenal. Classical Review. Oxford, n. 1, p. 243, 1887. WALLACE-HADRILL, A. Patronage in Roman Society: from Republic to Empire. In WALLACE-HADRILL, A. (Ed.). Patronage in Ancient Society. London-New York: Routledge, 1989, p. 63-87. 
VITORINO, Mơnica Costa. La clientelle dans les Satires de Juvénal. Classica, São Paulo, 15/16, p. 131-142, 2002/2003.

RÉSUMÉ: Cet article a pour but l'analyse des rapports entre patrons et clients, un thème largement traité dans les satires de Juvénal, ce qui le rend un instrument extrèmement important pour l'étude du portrait de la société romaine fourni par le satirique.

MOTS-CLÉS: Juvénal; satire; client; patron; société romaine. 